\title{
COMPOSICIÓN QUÍMICA DEL ACEITE ESENCIAL DE HOJAS De Myrcianthes rhopaloides (Kunt) McVaugh (MYRTACEAE)
}

Fecha de recepción: 26 de enero de 2016 • Fecha de Evaluación: 15 de marzo 2016 • Fecha de aceptación: 17 de marzo de 2016

\section{CHEMICAL COMPOSITION OF THE ESSENTIAL OIL leaves Myrcianthes rhopaloides (Kunt) McVaugh (MYRTACEAE).}

Diego Alejandro Silva Carrero1, Javier Andrés Matulevich Pelaez^, Beatriz Ofelia Devia Castillo²

\section{RESUMEN}

A partir de hojas frescas de Myrcianthes rhopaloides (Kunt) McVaugh, (arrayán) fueron extraídos los aceites esenciales, por destilación con arrastre de vapor. La caracterización del aceite se llevó a cabo por cromatografía de gases acoplada a espectrometría de masas (CG-EM), comparación de los índices de retención y los espectros de masas reportados en la literatura. Un $72.5 \%$ de los compuestos fueron identificados, entre los que se encuentran 11 monoterpenos (27.5\%), 9 monoterpenos oxigenados (22.5 \%), 7 sesquiterpenos (17.5\%) y 2 sesquiterpenos oxigenados (5\%) siendo el citronelal el monoterpeno de mayor abundancia en el aceite con un $27.3 \%$ dentro de la composición del aceite. Estos resultados contribuyen al conocimiento de los componentes volátiles en especies nativas y constituyen un aporte a la fitoquímica de Myrcianthes rhopaloides.

Palabras clave: Citronelal, CG-EM, Monoterpenos, Arrayan, Sesquiterpenos.

1 Universidad Distrital Francisco José de Caldas, Laboratorio de Productos Naturales Vegetales, Bogotá, Colombia.

2 Universidad Distrital Francisco José de Caldas, Laboratorio Colorantes Naturales, Bogotá, Colombia.

*Autor Corresponsal. E-mail: jamatulevichp@udistrital.edu.co 


\section{ABSTRACT}

From the fresh leaves of Myrcianthes rhopaloides (Kunt) McVaugh (Arrayán) were extracted the essentials oils by distillations with steam stripping. For oil characterization was applied gas chromatography-mass spectrometry (GC-MS) and the results were compared with retention indices and mass spectra reported in the literature. $72.5 \%$ of the compounds were identified, among which are: 11 monoterpenes (27.5\%), 9 oxygenated monoterpenes (22.5\%), 7 sesquiterpenes (17.5\%) and 2 oxygenated sesquiterpenes (5\%). The monoterpene citronellal is the most abundant (27.3\%). These results contribute to knowledge of the volatile components in native plant species and to the plant chemistry of Myrcianthes rhopaloides.

Key words: Citronellal, GC-MS, Monoterpenes, Arrayan, Sesquiterpenes.

\section{INTRODUCCIÓN}

La familia Myrtaceae está compuesta por 133 géneros y alrededor de 3900 especies, que se distribuyen en un alto porcentaje en las regiones tropicales y subtropicales de Australia y Suramérica desde los 2500 a 3200 m.s.n.m. (Wielgorskaya, 1995). El género Myrcianthes ha sido ampliamente utilizado en la medicina popular, es el caso de Myrcianthes pseudomato la cual es reconocida por su capacidad antimicrobiana (Demo et al,2002), Myrcianthes cisplatensis utilizada para el lavado y cicatrización de heridas, además de ser conocidos sus efectos en las afecciones bronquiales y de pulmones (Apel et al,2006).

En cuanto a la composición química del aceite esencial del genero Myrcianthes se referencian diversos compuestos clasificados en su mayoría como monoterpenos y sesquiterpenos, es el caso de $M$. pseudomato de Argentina, donde se reportó la presencia de 1-8 cineol y $\beta$-cariofileno (Demo et al, 2002), para M. coquimbensis, nativa de chile fueron identificados como compuestos mayoritarios limoneno, $\alpha$-pineno y carvona (Turker et al, 2002), en el caso de $M$, osteomeloides originaria de Bolivia se presentan como compuestos mayoritarios el 1-8 cineol, $\alpha$-pineno y linalol (Arze et al,2005).
Myrcianthes rhopaloides es un árbol de tronco cilíndrico color rojo marrón y copa de forma regular que puede alcanzar una altura hasta de $15 \mathrm{~m}$; sus hojas son simples opuestas con limbo ovalado color verde oscuro y envés verde amarillento, con una nervadura central muy prominente de 4 a $8 \mathrm{~cm}$ de longitud. (Cole et al, 1993). Esta especie se conoce con el nombre común de arrayan, sus usos alimenticios son muy conocidos, además de su capacidad medicinal en el control de la diabetes. (Lizcano et al, 2009).

En cuanto a la composición química del aceite esencial de Myrcianthes rhopaloides se pueden citar algunos estudios como los realizados sobre especies distribuidas en Ecuador en las cuales se determinó la presencia de geranial (33.7\%) neral (25\%), $\alpha$-pineno (6.9\%) y $\beta$-pineno (9.0\%), como componentes mayoritarios (Malagon, Vila, Iglesias, Zaragoza, Cañigueral., 2008); mientras para especies con distribución en Monte Verde-Costa Rica se determinó linalool (17.7\%), $\boldsymbol{\alpha}$-cadinol (14.4\%) y espatulenol (11.1\%) (Cole et al; 2008). En este trabajo se presenta la caracterización química del aceite esencial en M.rhopaloides con distribución en Macheta-Colombia. 


\section{MATERIALES Y MÉTODOS}

\section{Recolección y clasificación del material vegetal}

El material vegetal fue recolectado en el municipio de Macheta, Cundinamarca (Coordenadas geográficas: 05o 5' 42" Latitud norte - 730 38' 33" Longitud oeste) a $6 \mathrm{~km}$ del casco urbano en la vereda Quebrada Honda en el mes de Octubre de 2012. Una muestra testigo fue enviada al Herbario Nacional Colombiano para su determinación taxonómica la cual fue clasificada como Myrcianthes rhopaloides (Kunt) McVaugh bajo el número de colección COL573964.

\section{Obtención del aceite esencial y determinación de la composición química por CG-EM}

La obtención del aceite se realizó mediante la extracción con arrastre de vapor a partir de $500 \mathrm{~g}$ de hojas frescas y reducidas de tamaño, durante 4 horas. El producto de la extracción fue secado con sulfato de sodio anhidro, presentando un rendimiento del $0.28 \%$ y una masa de 1,40 $\mathrm{g}$.

La determinación de la composición química relativa del aceite esencial se realizó por CG-EM en un equipo SHIMADZU OP2010 plus, empleando una columna capilar de sílice fundida, HP-5MS (J \& W Scientific, Folsom, CA, EE.UU.) de $60 \mathrm{~m} \times$ $0.25 \mathrm{~mm} \times 0.25 \mu \mathrm{m}$, con fase estacionaria 95\% dimetilpolisiloxano y $5 \%$ de fenilo. La programación de temperatura del horno fue de $40^{\circ} \mathrm{C}$ (5 min) con incremento de $4{ }^{\circ} \mathrm{C} / \mathrm{min}$, hasta $160^{\circ} \mathrm{C}(0 \mathrm{~min})$ posterior a este se aumentó en $2.5^{\circ} \mathrm{C} / \mathrm{min}$, hasta $220^{\circ} \mathrm{C}$ (0 min) y finalmente $8{ }^{\circ} \mathrm{C} / \mathrm{min}$, hasta $280^{\circ} \mathrm{C}$ (4 min). Los espectros de masas se obtuvieron por impacto electrónico (IE) con 70 eV de energía. Las temperaturas de la cámara de ionización y de la línea de transferencia fueron de 230 y $325{ }^{\circ} \mathrm{C}$, respectivamente. El gas de arrastre utilizado fue helio (grado 5.0), con flujo constante de $1,0 \mathrm{~mL} / \mathrm{min}$. Los índices IR se calcularon teniendo en cuenta los tiempos de retención de una serie homologa de patrones de hidrocarburos desde $\mathrm{C}_{8}$ hasta $\mathrm{C}_{20}$, analizados bajo las mismas condiciones que el aceite esencial. La identificación de los componentes presentes en el aceite esencial de la especie Myrcianthes rhopaloides se realizó mediante la comparación de los índices de retención reportados en la literatura (Goodner, 2007; Adams 2007) y los espectros de masas almacenados en la librería NIST 08.

\section{RESULTADOS Y DISCUSIÓN}

Se presentan a continuación (tabla 1) los compuestos identificados de la especie Myrcianthes rhopaloides por CG-EM, a través de la comparación de sus índices de retención y los espectros de masas de la librería NIST 08; se reportaron los compuestos que por comparación presentaban más de un $90 \%$ de coincidencia con el espectro de la librería, también se presentan las cantidades relativas que corresponden al porcentaje de abundancia de cada componente dentro del aceite esencial.

Se logró identificar 29 compuestos (72.5\%) de la composición total del aceite; de los cuales fueron identificados 11 monoterpenos (27.5\%), 9 monoterpenos oxigenados (22.5\%), 7 sesquiterpenos (17.5\%) y 2 sesquiterpenos oxigenados (5\%).

Entre los monoterpenos se determinó $\alpha$-pineno y $\beta$-pineno con porcentajes de área de (4.20\%) y $(4.16 \%)$, respectivamente, $\beta$-citronelol con un porcentaje de área de (15.49\%), $\beta$-mirceno con un porcentaje de área (17.70\%), citronelal con un porcentaje de área de (27.34\%) siendo este último el compuesto con mayor porcentaje dentro de la composición del aceite. Además se identificaron otros compuestos con menor porcentaje como el $\alpha$-citral $(1,43 \%)$, ácido citronélico $(0,77 \%)$ y acetato de citronelol (1.92\%). En cuanto a los sesquiterpenos de mayor composición dentro del aceite se logró identificar $\beta$-elemeno (1.18\%), $\alpha$-farmeseno (2.23\%), y cariofileno (2.45\%). 
Tabla 1. Composición química relativa del aceite esencial obtenido en la columna RTX-5.

\begin{tabular}{|c|c|c|c|c|c|c|}
\hline No. Señal & $t_{\mathrm{RX}}$ & IR cal. & IR Ref & $\begin{array}{l}\text { Nombre del } \\
\text { compuesto }\end{array}$ & $\%$ & * \\
\hline 1 & 19.976 & 940.68 & 939 & $\alpha$-Pineno & 4.20 & M \\
\hline 2 & 21.996 & 984.88 & 980 & $\beta$-Pineno & 4.16 & $M$ \\
\hline 3 & 22.347 & 992.14 & 991 & $\beta$-Mirceno & 17.70 & M \\
\hline 4 & 23.941 & 1030.76 & 1026 & p-Cimeno & 0.31 & M \\
\hline 5 & 24.169 & 1036.39 & 1031 & d-Limoneno & 1.07 & $M$ \\
\hline 6 & 24.265 & 1038.74 & 1031 & $\beta$-felandreno & 0.99 & $M$ \\
\hline 7 & 24.741 & 1050.28 & 1050 & $\beta$-Ocimeno & 0.45 & M \\
\hline 8 & 25.357 & 1064.89 & 1062 & $V$ - terpineno & 0.56 & M \\
\hline 9 & 26.917 & 1100.44 & 1098 & $\beta$-Linalol & 0.14 & M \\
\hline 10 & 29.094 & 1158.15 & 1153 & Citronelal & 27.34 & M \\
\hline 11 & 29.233 & 1161.69 & 1156 & Isopulegol & 2.33 & M \\
\hline 12 & 29.562 & 1169.99 & 1168 & Neoisopulegol & 6.59 & M \\
\hline 13 & 29.938 & 1179.37 & 1164 & Isomentona & 0.37 & M \\
\hline 14 & 30.342 & 1182.32 & 1173 & Mentol & 0.21 & M \\
\hline 15 & 31.837 & 1220.17 & 1228 & Rodinol & 15.49 & M \\
\hline 16 & 32.653 & 1265.59 & 1255 & Geraniol & 0.46 & M \\
\hline 17 & 33.333 & 1280.69 & 1270 & Geranial & 1.43 & M \\
\hline 18 & 36.085 & 1349.01 & 1354 & $\begin{array}{c}\text { Propanoato de } \\
\text { Citronelol }\end{array}$ & 1.92 & M \\
\hline 19 & 36.434 & 1358.07 & 1351 & $\alpha$-Cubebeno & 0.34 & M \\
\hline 20 & 37.563 & 1390.44 & 1376 & Copaeno & 0.47 & M \\
\hline 21 & 37.945 & 1400.93 & 1391 & $\beta$-elemeno & 1.18 & $S$ \\
\hline 22 & 39.264 & 1428.47 & 1418 & Cariofileno & 2.45 & $S$ \\
\hline 23 & 40.56 & 1464.14 & 1454 & Humuleno & 1.11 & $S$ \\
\hline 24 & 41.453 & 1488.07 & 1480 & Germacreno D & 0.49 & $S$ \\
\hline 25 & 41.788 & 1503.79 & 1508 & $\alpha$-Farneseno & 2.23 & $S$ \\
\hline 26 & 42.657 & 1512.18 & 1503 & $V$-cadineno & 0.44 & $S$ \\
\hline 27 & 45.338 & 1572.88 & 1581 & Oxido de cariofileno & 0.20 & $S$ \\
\hline 28 & 47.913 & 1656.14 & 1653 & $\alpha$-Cadinol & 0.20 & $S$ \\
\hline 29 & 48.146 & 1670.06 & 1652 & Eudesmol & 0.59 & $S$ \\
\hline
\end{tabular}

$\mathrm{t}_{\mathrm{RX}}$ : Tiempo de retención columna RTX-5; IR cal: Índice de retención calculado; \%: Porcentaje relativo en el aceite esencial; * Monoterpenos (M), Sesquiterpenos (S), IR Ref: Índice de retención de referencia. Columna DB5. 
El aceite esencial se obtuvo con un rendimiento del $0.28 \%$, lo cual es coherente con lo descrito por Goralaka et al, (1996) donde el contenido del aceite esencial de una planta puede variar en función de la época del año en cual fue colectado el material vegetal, su forma de desenvolvimiento, factores biológicos, localización y factores ecológicos (Oliveira et al, 2005). Este porcentaje obtenido es característico de la familia Mirtácea; familia que se caracteriza por presentar alto rendimiento en aceite esencial teniendo en cuenta la alta capacidad de biosíntesis de compuestos derivados de feníl propanóides, hidrocarburos alifáticos, compuestos terpénicos y compuestos oxigenados en hojas (Nakamura et al, 2010; Cole et al, 2007; Padovan et al, 2014).

Al comparar la composición de los componentes volátiles con otras especies del género Myrcianthes, se evidencia gran variabilidad entre porcentajes de área y componentes químicos del aceite esencial. Para Myrcianthes cisplatensis, Myrcianthes gigantea y Myrcianthes pugens, originarias del sur de Brasil se reportaron monoterpenos hidrocarbonados como limoneno en $M$. cisplatensis con un porcentaje de área de (10.9\%) y para M. gigantea el $\alpha$-pineno con un porcentaje de área de (4.5\%). Algunos monoterpenos oxigenados como el 1,8-Cineol (29.8\%) para M. cisplatensis; para M. pugens $(2.1 \%)$ y para M. gigantea se reporto una abundancia del 1.4\% (Apel et al, 2006). Además, se han reportado algunos sesquiterpenos hidrocarbonados como $\beta$-cariofileno con un porcentaje de área de $(10,8 \%)$ para $M$. cisplatensis y $(10.1 \%)$ para M. pugens y $\alpha$-copaeno (2.6\%) para M. gigantea. En cuanto a sesquiterpenos oxigenados como el Guaiol se ha reportado en $M$. cisplatensis con un porcentaje del $4.9 \%$ y espatulenol en $M$. gigantea (28.9\%) y en M. pugens con una abundancia del 9.7\% (Apel et al., 2006). Lo anterior permite resaltar componentes comunes entre los cuales se encuentran el $\alpha$ pineno y el $\beta$-cariofileno presentes tanto en las especies originarias del Brasil como en la especie M. rhopaloides de Colombia, compuestos que reafirman la hipótesis de ser posibles taxones químicos del género. (Padovan et al, 2014; Cole et al, 2008; Malagon et al, 2003).

La relación de los componentes identificados en el aceite esencial de Myrciathes rhopaloides, de Machetá, Colombia y el aceite esencial extraído de Myrcianthes rhopaloides de Monteverde; Costa Rica (Cole et al, 2008), indica como compuestos comunes para las dos especies el $\alpha$ - y $\beta$-pineno. En cuanto a los monoterpenos mayoritarios existen diferencias marcadas ya que para el caso del aceite extraído en este estudio se identificó el citronelal como compuesto mayoritario, mientras que en el estudio de Myrcianthes rhopaloides originario de Monteverde se reporta el linalol como monoterpeno mayoritario. El aceite esencial de M. rhopaloides originario de Ecuador reporta como compuesto mayoritario al geraniol, mientras que en el aceite extraído de la especie de Colombia es un componente minoritario. Las relaciones anteriores están de acuerdo con lo reportado por Cole (2008) donde se afirma que existen variaciones quimiotaxonómicas dentro de la especie Myrciathes rhopaloides.

Es importante resaltar las actividades encontradas para algunos de los compuestos presentes en el aceite esencial de Myrcianthes rhopaliodes; siendo así la actividad antiinflamatoria reportada por Manzano et al, (2011) para los compuestos $\beta$ -elemeno, $\alpha$-farmeseno, y cariofileno. Algunos de los componentes mayoritarios que están presentes en el aceite esencial de Myrcianthes rhopaloides, se han utilizado en otras investigaciones reportando diversas actividades, o como precursores de síntesis, así por ejemplo la síntesis de mentol a partir de citronelal utilizando como catalizadores sulfato de níquel y circonio (Barrales et al, 2011), siendo el mentol un componente ampliamente utilizado en la industria farmacéutica, de cosméticos, y alimenticia; 
para el $\beta$-Mirceno, se han reportado actividades anti-inflamatoria y anti-catabólica (Rufino et al, 2015), También es reportado por (Gomes-Carneiro et al, 2005) la evaluación genotoxicologica para los compuestos $\beta$-mirceno, $\alpha$-terpineno y $\boldsymbol{\alpha}$-pineno $(+)$ frente a Salmonella/microsome, dando positivo frente a este microorganismo.

\section{AGRADECIMIENTOS}

Los autores expresan su agradecimiento a la Universidad Distrital Francisco José de Caldas, a Luz Stella Villalba del laboratorio de microbiología de la Biblioteca Nacional de Colombia, quienes dieron su apoyo a la investigación en diferentes instancias.

\section{REFERENCIAS}

1. Adams R, 2007, Identification of essential oil components by gas chromatography / mass spectroscopy. Allured Publishing Corporation. USA, 468p.

2. Apel M, Sobral M, Henriques A, 2006, Composição química do óleo volátil de Myrcianthes nativas da região sul do Brasil, Revista Brasileira de Farmacognosia, 16 (3):402-407

3. Arze JBL, Jean FI, Gagnon H, Collin G, Garneau F, Pichette A, 2005, Essential oils from Bolivia. VII. Myrtaceae: Myrcianthes osteomeloides (Rusby) McVaugh and Myrcianthes pseudomato (Legrand) McVaugh. Journal of Essential Oil Research, 17: 64-65.

4. Barrales C, Tamayo V, Santiago S, Viveros T, 2011, One pot synthesis of menthol from $( \pm)$-citronellal on nickel sulfated zirconia catalysts, Catalysis Today, 172:21-26.

5. Cole R, Haber W, Lawton R, Setzer W, 2008, Leaf Essential Oil Composition of Three Species of Myrcianthes from Monteverde, Costa Rica, Chemystry \& Biodiversity. 5:1327-1334. 
6. Cole R, Haber W, Setzer W, 2007, Chemical composition of essential oils of seven species of Eugenia from Monteverde, Costa Rica, Biochemical Systematics and Ecology 35:887-886.

7. Demo S M, Olivia M, Zunino M, Lopez M, \& Zyngadlo J, 2002, Aromatic Plants from Yungas. Part IV: Composition and Antimicrobial Activity of Myrcianthes pseudo-mato Essential Oil. Pharmaceutical Biology, 40(7):481-484.

8. Gentry, A. 1993.A Field Guide to the Families and Genera of Woody Plants of Northwest South America (Colombia, Ecuador, Perú). Pp 646

9. Gomes-Carneiro MR, Marcias ES, Felzenswalb I, Paumgartten FJR, 2005, Evaluation of bmyrcene, a-terpinene and (+)- and (-)-a-pinene in the Salmonella/microsome assay, Journal Food and Chemical Toxicology 43: 247-252.

10

Goodner K, 2007, Practical retention index models of OV-101, DB-1, DB-5, and DB-Wax for flavor and fragrance compound LWT-Food Science and Technology, 41:951-958
11. Goralaka JLR, Shumaker MA, Langenheim JH, 1996, Variation in chemical and physical proprieties during leaf development in California Bay tree (Umbellularia californica): predictions regarding palatalability for deer. Biochem Syst Ecol. 24: 93-103.

12. Lizcano A, Torres M, Vergara J, 2009, Evaluacion de la actividad antimicrobiana de los extractos etanolicos y aceites esenciales de las especies vegetales Valeriana pilosa, Hesperomeles ferruginea, Myrcianthes rhopaloides, y Passiflora manicata, frente a microorganismo patógenos. Pérez-Arbelaezia Revista José Celestino Mutis. 19:163-187

13. Malagon $O$, Vila R, Iglesias J, Zaragoza T, Cañigueral S, 2003, Composition of the essential oils of four medicinal plants from Ecuador, Flavour and Fragrance Journal, 18:527-531.

14. Manzano P, Miranda M, Gutierrez y, Garcia G, Orellana T, Orellana A. 2011, Efecto antiinflamatorio y antimicótico del extracto alcoholico y composición química del aceite esencial de 
Conyza bonariensis (L.) Cronquist (Canilla de venado), Revista Cubana de plantas medicinales, 16(1):13-23.

15. Matulevich J, Gil E, 2014, Composición química del aceite esencial de hojas de Bejaria resinosa (Ericaceae). Revista Facultad de Ciencias Básicas. 10(2): 204-209.

16. Nakamura M, Monteiro S, Bizarri C, Siani A, Ramos M, 2010, Essential oils of four Myrtaceae species from the Brazilian southeast, Biochemical Systematics and Ecology 38:1170-1175.

17. Oliveira RN, Dias IJM, Câmara CAG, 2005. Estudo comparativo do óleo essencial de Eugenia punicifolia (HBK) DC de diferentes localidades de Pernambuco. Revista Brasilera de Farmacognosia,15: 39-43.

18. Padovan A, Keszei A, Kulheim C, Forley W, 2014, The evolution of foliar terpene diversity in Myrtaceae, Phytochemistry Reviews, 13:695:716.

19. Parra C, 2012, Una nueva especie de Myrcianthes (MYRTACEAE) de Colombia. Caldasia 34(2): 277-282.
20. Rufino A, Ribeiro $m$, Sousa C, Judas F, Salgueiro L, Cavaleiro C, Ferreira A, (2015), Evaluation of the anti-inflammatory, anti-catabolic and pro-anabolic effects of E-caryophyllene, myrcene and limonene in a cell model of osteoarthritis, European Journal of Pharmacology, 750: 141-150.

21. Tuerker AO, Maciarello MJ, Landrum LR, (2002), Volatile leaf oil of Myrcianthes coquimbensis (Barnoud) Landrum et Grifo (Myrtaceae) of Chile, Journal of Essential Oil Research, 14:40-41

22. Wielgorskaya T, 1995. Dictionary of generic names of seed plants. Columbia University Press, New York. (2):570-575. 